INTERNACIONAL

\title{
Educación en derechos humanos como política pública: La experiencia de la Coordinación de Educación en Derechos Humanos de la Secretaría Municipal de Derechos Humanos y Ciudadanía de la ciudad de São Paulo
}

\author{
Human Rights Education as a Public Policy: The experience of São Paulo's \\ Municipal Secretariat Coordinating Human Rights Education
}

Eduardo C. B. BitTAR

Universidad de São Paulo, Brasil

\begin{abstract}
RESUMEN Este artículo es un informe sobre las experiencias en gestión pública y coordinación de políticas públicas municipales de educación en derechos humanos, en el contexto de la creación de la primera Secretaría Municipal de Derechos Humanos y Ciudadanía (SMDHC) de la historia de la ciudad de São Paulo, Brasil. Este informe expone los principales resultados de cuatro años de trabajo de construcción y articulación de políticas, con vistas a un proceso que culmina en la publicación del Decreto Municipal 57.503/2016, que crea el Plan Municipal de Educación en Derechos Humanos de la ciudad de São Paulo. Fue así como, a lo largo de un período relativamente corto, y por medio de esfuerzos colectivos internos y externos, se construye la SMDHC en el marco municipal, con relevantes conquistas reales y simbólicas, y haciendo que la política municipal de derechos humanos no solamente llenase una laguna en la historia del municipio, sino que también presentara políticas transversales, innovadoras, valientes y desafiantes en el «corazón simbólico» de la ciudad de São Paulo.
\end{abstract}

PALABRAS CLAVE Educación en derechos humanos, políticas públicas, gestión pública.

ABSTRACT This article is an experience report about coordinating municipal public policies on human rights education, in the context of the creation of the Municipal Secretary of Human Rights and Citizenship (SMDHC) of the City of São Paulo, Brazil. This paper presents the main results of four years of construction work and articulation of policies, with a view to a process that culminates in the publication of Municipal Law $57.503 / 2016$, that creates the Municipal Plan of Education in Human Rights of the City of 
São Paulo. Thus, over a relatively short period of time, and through internal and external collective efforts, the SMDHC is built in the municipal framework, with significant real and symbolic achievements, and making municipal human rights policy not only fill a gap in the history of the Municipality, but also present transversal, innovative, courageous and challenging policies in the «symbolic heart» of the City of São Paulo.

KEYWORDS Human rights education, public policy; public administration.

\section{La creación de la Secretaría Municipal de Derechos Humanos}

En este artículo se encontrará un relato de experiencia que es resultado de un trabajo cuatro años de la Coordinación de Políticas Públicas de Educación en Derechos Humanos en la ciudad de São Paulo. El artículo está dividido en cuatro partes: la primera está dedicada a presentar el proceso de creación de la Secretaría Municipal de Derechos Humanos (SMDHC), la segunda a presentar la política municipal de educación en derechos humanos, la tercera a presentar los resultados cuantitativos del periodo, y finalmente una conclusión.

Todo empezó con el proceso de creación de la primera Secretaría Municipal de Derechos Humanos en la historia de la ciudad de São Paulo (Decreto Municipal 53.685, del 1 de enero de 2013; Ley Municipal 15.764, del 27 de mayo de 2013). En una ciudad cuya historia se remonta, en su período fundacional, a la población de São Paulo de Paraitinga, el 25 de enero de 1554, esto no es algo simple de explicar. Se puede abordar desde diversas perspectivas, con enfoques que enfaticen éste o aquel aspecto. Por eso mismo, la narrativa a utilizar para la creación de este informe de experiencia buscará destacar los principales resultados de las políticas públicas municipales, con énfasis en el área de educación en derechos humanos, así como presentar las producciones de la época de la forma más consolidada y sistematizada posible.

Se dio inició así, y en medio de discusiones de gestión pública, al proceso de implementación de políticas públicas de educación en derechos humanos partiendo de una clara comprensión y concepción sobre el significado de «educación en derechos humanos» $(\mathrm{EDH})^{1}$, a través de la Coordinación de Educación en Derechos Humanos de la SMDHC (artículo 242, inciso 2 de la Ley Municipal 15.764). En su misión institucional, se definió como plan de trabajo y directrices de acción colectiva, conforme

1. En la lapidaria definición de Maria Victória Benevides, que la caracteriza de la siguiente forma: «Para ello, se debe partir de tres puntos esenciales: primero, es una educación de naturaleza permanente, continuada y global. Segundo, es una educación necesariamente volcada hacia el cambio. Y tercero, es una formación en valores, para alcanzar corazones y mentes, y no sólo instrucción, meramente transmisora de conocimientos» (Benevides, 2009: 323). Esta y todas las traducciones en este artículo son nuestras. 
consta en la página electrónica del SMDHC (enlace de la Coordinación de Educación en Derechos Humanos) ${ }^{2}$.

\section{La política municipal de educación en derechos humanos}

La determinación inicial que movilizó los esfuerzos por convertir las metodologías de la educación en derechos humanos - sus objetivos, sus contornos y marcos conceptuales ${ }^{3}$ - en iniciativas, acciones, proyectos y formaciones fue la percepción de que la escuela contemporánea se encuentra entrecortada por fenómenos que mutilan la propia consistencia de la sociedad actual, en especial frente a la violencia, lo que perjudica el ejercicio del derecho a la educación (Schilling, 2014: 16). Para hacer frente a este tipo de desafío, el punto de partida para la construcción de proyectos, iniciativas y consensos en torno al tema de la educación en derechos humanos fue el Plan Nacional de Educación en Derechos Humanos (PNEDH) de 2006, que desempeñó el papel de documento rector de todas las políticas del área, desde la idea de una actuación en que la formación humana, crítica, reflexiva y pluralista fuese el objetivo metodológico de la perspectiva y abordaje de la temática en derechos humanos.

A partir de esta definición, fue posible superar rápidamente la cultura de iniciativas aisladas» de educación en derechos humanos, para ir construyendo una «cultura de políticas públicas» (Bucci, 2002: 249) sistemáticas, continuas y enraizadas en el trabajo de diversos actores esparcidos por los territorios de la ciudad. Pero, construir los proyectos con el y los otros, en los espacios colectivos de decisión y deliberación, respondiendo a presiones internas y externas, $y$, progresivamente, con la capacidad de entrever sus futuros pasos, fue algo desarrollado, en el caos de lo cotidiano, con vistas a la atribución de un carácter permanente, territorial, autónomo e independiente de las acciones, iniciativas y políticas públicas.

Los desafíos no fueron pocos a lo largo del tiempo, pues implicaban demandas simultáneas en torno a cuestiones ligadas a la gestión administrativa y a la implementación de políticas. Y, de hecho, se exigió un trabajo muy dedicado y proactivo de todos los equipos que compusieron la Coordinación de Educación en Derechos Humanos, espacio creado para trabajar la educación en derechos humanos, en la que se encontró la necesidad de ejercer las funciones profesionales con una polivalencia que exigió un máximo de capacidades, habilidades y conocimientos de cada uno.

Por eso, los caminos de escucha y valorización de experiencias de los territorios

2. El sitio web de la Coordenação de Educação em Direitos, SMDCH, de la Alcaldía de São Paulo se encuentra disponible en http://bit.ly/2ueOokF.

3. «Así, cuando en los estudios contemporáneos se quiere desvelar un concepto adecuado de educación en derechos humanos, se debe enfatizar que educar sólo tiene sentido mientras se valorice la dignidad de la persona humana como fundamento de la convivencia pluralista, abierta, democrática, inclusiva, participativa y no violenta» (Bittar, 2016: 165). 
fueron elementos valiosos en el proceso y en los resultados de las políticas, por lo que se evitaron construcciones «de arriba abajo», propias de jerarquías institucionales, y sí se usaron las «construcciones en conjunto con» para la búsqueda de participación de los actores involucrados y cambios recíprocos permanentes con los territorios afectados por las acciones. Al final, la concepción de educación en derechos humanos, a partir de la cual se derivaron todas las iniciativas, parte de un modelo teórico en que estas dimensiones son innegociables, pues tiene como objetivo trabajar con «sujetos de derechos» ${ }^{4}$, considerando que la experiencia del otro está imbuida en el modo de ser del otro, sea del estudiante, sea del gestor, sea del educador:

Por qué no aprovechar la experiencia que tienen los alumnos de vivir en áreas de la ciudad descuidadas por el poder público para discutir, por ejemplo, la contaminación de los riachuelos y las corrientes, los bajos niveles de bienestar de las poblaciones, los vertederos y los riesgos que provocan para la salud de la gente (Freire, 2013: 31-32).

La garantía de que la educación en derechos humanos sería, de hecho, implementada y ejecutada, y no quedaría como parte superficial o residual de las políticas municipales, emergió de la propia postura asumida por el Programa de Metas del Ayuntamiento de São Paulo, en el que se inscribieron varios objetivos por parte de la SMDHC, entre ellos se destacaron la meta 63 («implementar la educación en derechos humanos en la red municipal de enseñanza») y la meta 39 («capacitar seis mil agentes de la Guardia Civil Metropolitana en derechos humanos y dos mil en mediación de conflictos»), considerado el propio programa de transparencia y prestación de cuentas del desempeño de las políticas públicas, frente a la población y los electores.

Esto porque se era consciente de la importancia de no paralizar en un momento decisivo, en que Brasil cuenta con un importante trabajo de incorporación de la educación en derechos humanos ya realizado, si además se considera que el Programa Mundial de Educación en Derechos Humanos se encuentra actualmente en su tercera fase de ejecución (2015-2019). En especial, la ciudad de São Paulo tiene un trabajo importante, que puede servir de referencia para otras ciudades del país y del mundo, para tantas otras capitales mundiales con los mismos problemas y dimensiones, por ser la principal ciudad brasileña en número de población y en producción económica, además de su importancia cosmopolita.

4. «Hacer que el educador se perciba sujeto de derechos y de deberes, se responsabilice como perteneciente a una comunidad de socios de derecho, y se haga consciente de todo el poder de su empoderamiento en esta condición, son los efectos buscados en el perfil de formación en educación de derechos humanos» Bittar, 2016: 168). 


\section{Los legados de las políticas públicas de educación en derechos humanos}

Los legados de este proceso constructivo pasan por incorporar marcas visibles e invisibles de derechos humanos en la ciudad de São Paulo. Entre las marcas visibles se encuentran aquellas realizadas con técnicas artísticas en los muros de las calles de la ciudad, a lo largo de los cuatro festivales Ciudadanía en las Calles; durante y después de conciertos al aire libre, con gran asistencia de público, en el Auditorio del Parque de Ibirapuera; el cambio de nombres en vías públicas dentro del proyecto Calles de Memoria, en especial el cambio del nombre del viaducto («elevado Presidente Arthur Costa e Silva» para «elevado João Goulart»), así como del Monumento a los 436 Muertos y Desaparecidos Políticos, proyectado por Ricardo Ohtake e instalado en el parque de Ibirapuera; de varios equipamientos públicos esparcidos por diversos territorios de la ciudad, en centros de educación en derechos humanos, centros de ciudadanía de emigrantes y de centros de ciudadanía LGBT. Entre las marcas invisibles se encuentra un trabajo de cambio de mentalidad, y, por tanto, de transformación de personas, en la formación de educación en derechos humanos, ya sea dentro o fuera de la SMDHC, pues se sabe que el primer esfuerzo en esta área tendría que haber sido exactamente el de «mirar hacia adentro» antes de «mirar hacia afuera».

Es importante citar, sin embargo, que la legitimidad del discurso de la educación en derechos humanos y su entrada en determinados espacios, institucionalizados o no institucionalizados, fue conquistada paulatinamente, superando resistencias, de un modo recoveco y con retrocesos, en ciertas agendas y niveles, muchas de las cuales fueron frustradas, $y$, por eso, los resultados se fueron consolidando en la medida en que crecía la capacidad de generar adhesión de los socios de ejecución de las políticas públicas, sea en el ámbito de Secretarías, sea en el ámbito de los territorios. Para la adhesión de los socios, participantes activos de las etapas de trabajo, en tareas específicas compartidas y dentro de espacios en consolidación, se utilizaron canales e instancias y se enfatizó especialmente en el proceso de construcción democrática, el cual de por sí sólo ejerce el papel de generar aprendizaje colectivo como proceso participativo dentro de una concepción de democracia participativa ${ }^{5}$, y no únicamente en resultados, tomados como productos finales de las acciones, proyectos y políticas públicas.

Por eso mismo, un aspecto decisivo en la generación de resultados de escala y de impacto fue la institucionalización de espacios e instancias colectivas de construcción de las políticas públicas, tales como el Consejo de Educación en Derechos Hu-

5. «Por eso, la teoría del discurso considera impensable la democracia sin derechos humanos, y viceversa [...]. Así es que el desarrollo de una cultura social fundada en la amplia perspectiva de los derechos humanos tiene un papel central en la propia construcción de la democracia deliberativa y participativa» (Bittar, 2013: 402). 
manos (CEDH) y, enseguida, el Comité Municipal de Educación en Derechos Humanos (CMEDH), el Grupo de Trabajo Intersecretarial del Festival Entre Todos (GTIE), el Grupo de Trabajo Intersecretarial de Educación en Derechos Humanos de la Red Municipal de Enseñanza (GTI-EDH), el Núcleo de Educación en Derechos Humanos de la Red Municipal de Enseñanza (NEDH), compuesto por las trece Direcciones Regionales de la Red Municipal de Enseñanza, y el Grupo de Trabajo de los Centros de Educación en Derechos Humanos (GT-CEDH central y GT-CEDH locales).

Así, a lo largo de la época, y sabiéndose que el trabajo diario es mucho más costoso y difícil de lo que muestra la narrativa sobre los datos generados, determinadas marcas centrales se consolidaron como principales resultados del área, con conquistas simbólicas y reales en el campo de la $\mathrm{EDH}$, a saber:

- Formación de educadores: 6.265 educadores formados en temas de educación en derechos humanos, a través de varias estrategias, tales como la oferta de especializaciones de la UNIFESP/UFABC (GDE, EDH-MEC-SECADi), la realización de dos Seminarios Municipales (evento centralizado en 2014 con 182 participantes y evento descentralizado en 2015, con 1.394 participantes), participación en cursos presenciales y a distancia, formaciones « $i$ Respetar Es Necesario!», conferencias, ruedas de interlocución y otras actividades.

- Formación de agentes de seguridad urbana: 6.420 agentes de la Guardia Civil Municipal capacitados en derechos humanos y 4.547 agentes capacitados en mediación de conflictos pasaron por ciclos de formación (trece temas de derechos humanos) en el Centro de Formación en Seguridad Urbana de la Guardia Civil Municipal (SMSU, SMDHC).

- Participación junto al Centro de Formación de la Guardia Civil Municipal para la promoción de los derechos humanos en la cultura institucional, a través de diversas estrategias, tales como: formalización de la sociedad ENAM/MJ, SMSU y SMDHC para formación en mediación de conflictos; reformulación del currículo del Centro de Formación en Seguridad Urbana (doblando el número de horas en el curso anual de práctica profesional (Estágio de Qualificação Profissional, EQP, de cuatro a ocho horas aula); propuestas de nuevas disciplinas en el currículo de formación continua de los cursos del Centro de Formación en Seguridad Urbana; alteración del decreto de contratación de 2.00o nuevos GCM; actuación junto al Consejo Académico del Centro de Formación en Seguridad Urbana; distribución de kits temáticos de Entretodos; preparación de materiales pedagógicos y fortalecimiento de la colección del Centro de Formación en Seguridad Urbana; realización de formaciones temáticas.

- Creación y realización anual de las ediciones de cuatro versiones del Premio Municipal de Educación en Derechos Humanos de la Red Municipal de En- 
señanza (2013-2016), el cual fomenta, incentiva y destaca más de cuarenta proyectos premiados, en las categorías de unidad escolar, profesor, estudiante, gremio estudiantil e iniciativas de las unidades educacionales en temas de educación en derechos humanos y de derechos humanos, provenientes de varios territorios, relativos a temas diversificados en derechos humanos (violencia, sustentabilidad, memoria y verdad, nutrición, racismo en la infancia, primera infancia, gestión democrática, movilidad urbana, racismo, igualdad, arte y cultura, diversidad, entre otros), de todas las trece Direcciones Regionales de Enseñanza de la ciudad.

- Creación del Premio de Derechos Humanos Don Paulo Evaristo Arns: por medio del Decreto 55.759/2014 y del Edicto 037/SMDHC/2015, en cuanto homenaje del Municipio de São Paulo a personas (físicas o jurídicas) destacadas en la lucha por la defensa y por la promoción de los derechos humanos, a través de homenaje regularmente concedido en evento público en el Día Internacional de los Derechos Humanos (10 de diciembre), se han homenajeado a tres personalidades públicas, con respeto a la alternancia de género en la deliberación de los nombres, en la lucha histórica por los derechos humanos en la ciudad de São Paulo (Frei Betto, Luiza Erundina y el padre Jaime Crowe).

- Creación del curso de formación de funcionarios públicos municipales: iniciada por la necesidad de formación del gestor público de la propia SMDHC, en sociedad con la Escuela Municipal de Administración de São Paulo (EMASP) y la Secretaría Municipal de Gestión (SMG), ejecutada por la Escuela de Gobierno, con metodologías de enseñanza innovadoras (aulas expositivas, charlas temáticas, vivencias de campo, cine-debates, discursos, conferencias, declaraciones de funcionarios), habiéndose formado y diplomado a 190 funcionarios en derechos humanos de dieciocho Secretarías Municipales diferentes, con matriz curricular, metodología y educadores para la formación de cursos permanentes para funcionarios públicos en temas de derechos humanos («Formación Fundamental en Derechos Humanos y Ciudadanía», 24 horas aula; «Formación en Derechos Humanos EAD», 90 horas-aula) ${ }^{6}$.

- Participación política e intervención en las decisiones sobre el Plan Municipal de Educación de la ciudad de São Paulo: garantía de previsión (SME, SMDHC) en el Plan Municipal de Educación (PME) (Ley Municipal 16.271, del 17 de septiembre de 2015), documento orientador de la actuación de la Secretaría Municipal de Educación, con dos directrices para la educación municipal sobre educación en derechos humanos, válidas para la definición de los caminos

6. El contenido de estos cursos están disponibles en http://bit.ly/2hKFc1x. 
de la educación para los próximos diez años: «Promoción de la educación en derechos humanos» $\mathrm{y}$ «Difusión de los principios de equidad, de la dignidad de la persona humana y del combate a cualquier forma de violencia».

- Creación del Plan Municipal de Educación en Derechos Humanos de la ciudad de São Paulo: producción participativa (que involucra a actores múltiples $\mathrm{y}$ varias etapas participativas, a lo largo de un año de trabajo de consultas), y sistematización final del texto del Plan Municipal de Educación en Derechos Humanos (PMEDH), a partir del texto inicial del Plan Nacional de Educación en Derechos Humanos (PNEDH), creado por Decreto Municipal 57.503, del 6 de diciembre de 2016, con vistas a comprobar la unidad y atribuir la fuerza de un marco normativo municipal a las políticas municipales de educación en derechos humanos, a través de siete ejes de organización (educación básica, educación superior, educación no formal, educación en derechos humanos en el servicio público, educación de profesionales de los sistemas de justicia y seguridad urbana, educación y medios de comunicación).

- Creación de cuatro centros de educación en derechos humanos: institucionalización de cuatro centros de educación en derechos humanos (CEDH) (SMDHC, SME/SDH-PR) en centros de educación unificada (CEU), los que actúan como polos de diseminación de la EDH en los siguientes contextos: zona norte (CEU Jardín Paulistano, colección João Eloi do Nascimento); zona sur (CEU Casa Blanca, colección Doña Lurdes-Maria Cecília de Luna); zona este (CEU São Rafael, colección Brayan Yamarico); zona oeste (CEU Pera Marmelo, colección Seu Souza). Estos centros tienen como pilares de actuación los siguientes ejes de trabajo: gestión democrática, articulación territorial, producción de material didáctico-pedagógico, formación de profesionales de la educación y, por fin, colecciones temáticas de educación en derechos humanos en las bibliotecas de los CEU (que reúnen, en total, más de 5.40o libros y materiales de derechos humanos). El proyecto fue semifinalista, de entre 346 proyectos inscritos de dieciséis países, del concurso Derechos Humanos en América Latina: ¡Impulsando la Transformación!, organizado y promovido por la entidad Ashoka Changemakers?.

- Promoción de festivales de cine: coordinación y realización de cuatro Festivales de Cortometrajes en Derechos Humanos «Entretodos» (SMDHC, SME, SMC, SPCINE), con realizaciones temáticas anuales (2013, «transformaciones»; 2014, «ciudadanía cultural»; 2015, "ciudad educadora»; 2016, «refugiados») junto con formaciones en audiovisual y derechos humanos que alcanzaron a

7. Información sobre el concurso disponible en http://bit.ly/2uFRqgU. 
2.062 personas y lograron un impacto general de público en las exposiciones estimado en 32.000 personas, quienes llegaron a puntos de exhibición (de cultura y de educación) esparcidos por los diversos territorios de São Paulo, en especial los espacios de las periferias (2013, 45 puntos; 2014, 57 puntos; 2015, 76 puntos; 2016, 20 puntos). En ellos se premió a veintidós directores de cine y productores culturales.

- Estructuración de instancias políticas participativas y democráticas: formación de instancias colectivas de deliberación sobre la política municipal de educación en derechos humanos, tales como el Grupo de Trabajo Intersecretarial de Educación en Derechos Humanos (GTiEDH, SME/SMDHC), el Núcleo de Educación en Derechos Humanos (NEDH, SME/SMDHC), el Grupo de Trabajo Intersecretarial de Entretodos (SMC, SME, SMDHC, SPCine), todos activos de 2013 a 2016, cuando crearon e institucionalizaron el Comité Municipal de Educación en Derechos Humanos (CMEDH).

- Promoción de seminarios de educación en derechos humanos: coordinación y realización de un Seminario Municipal de Seguridad Urbana y Promoción de los Derechos Humanos (SMDHC, SMSU) y dos Seminarios Municipales de Educación en Derechos Humanos (SMDHC y SME).

- Producción de materiales pedagógicos: desarrollo de materiales pedagógicos de calidad, libres, públicos y gratuitos para apoyar acciones formativas, que consideran los diversos públicos afectados (profesionales de la educación, funcionarios públicos municipales, guardias civiles municipales, público del área de cine y cultura), tales como: mil ejemplares del libro Primer Premio Municipal de Educación en Derechos Humanos. Informes de experiencia; los tres libros en formato PDF Segundo, Tercero y Cuarto Premio Municipal de Educación en Derechos Humanos. Informes de experiencia; cinco mil ejemplares del Plan Nacional de Educación en Derechos Humanos (PNEDH); mil ejemplares del material «iRespetar es necesario! Educación en derechos humanos»; cinco mil ejemplares del libro Sistematización de prácticas de la Educación Básica (organizado por Paulo César Carbonari); el libro Centros de educación en derechos humanos de la ciudad de São Paulo. Territorios, educación y ciudadanía; seis mil copias de los DVD temáticos «Entretodos» (Ancianos; Emigrantes; Juventud; Niños y adolescentes; Derecho a la memoria y a la verdad/Violencia urbana; LGBT; Medios de comunicación y derechos humanos/Trabajo decente; Derecho a la ciudad/Participación social; Población de las calles/Alcohol y drogas) ${ }^{8}$.

8. Todos los materiales se encuentran disponibles de forma abierta para uso y consulta en http:// portaledh.educapx.com. 
- Elaboración de dictamen pareceres: dictamen favorable a la inclusión, en el currículum escolar de la red municipal de enseñanza, de la temática «derechos humanos» en la enseñanza fundamental, por medio de proyecto de ley $261 / 11$ del Concejal Jamil Murad, a través de la Ley 16.493, de 18 de julio de 2016, que, en su artículo 1 dispone: «Queda incluido en las disciplinas de Historia/Geografía del currículum de la enseñanza fundamental de las escuelas de la red municipal de enseñanza, un tema específico para debate y comprensión de los derechos humanos».

- Creación del Comité Municipal de Educación en Derechos Humanos: creación, por medio de Decreto Municipal, del Comité Municipal de Educación en Derechos Humanos (CMEDH) de 2016, con la tarea de acompañar y monitorizar la implementación del Plan Municipal de Educación en Derechos Humanos y las políticas públicas municipales de educación en derechos humanos.

- Apoyo a la Política Municipal de Primera Infancia: colaboración en el desarrollo de la política municipal volcada en la primera infancia, con énfasis en la actuación del proceso de creación de la Fundación de Protección y Defensa del Consumidor Municipal, y en el desincentivar la publicidad infantil abusiva o engañosa, actuándose bajo consulta preparatoria del Decreto Municipal 56.871, del 15 de marzo de 2016.

- Desarrollo de normas legales para el desincentivo a la publicidad infantil: creación de previsión legal de la necesidad de que la Fundación de Protección y Defensor del Consumidor actúe para "participar en la elaboración y acompañamientos de políticas públicas de desestimulo de publicidad engañosa y abusiva, inclusive la dirigida a niños y adolescentes» (artículo 5, inciso 18), y la «promoción de actividades y eventos educativos, culturales y científicos y en la edición de material informativo relacionado con la educación, protección y defensa del consumidor, inclusive en campañas de prevención de publicidad engañosa y abusiva dirigida a niños y adolescentes» (artículo 17, inciso 2), por medio de las sugerencias de texto participativo en el momento de la formulación jurídica del texto del Decreto Municipal 56.871, que creó la figura del Defensor del Consumidor Paulistano.

\section{Conclusiones}

El esfuerzo mayor del desafío de hacer posible la educación en derechos humanos en la realidad de los estudios y trabajos de muchas personas en la ciudad de São Pablo fue entre 2013 y 2016. Desde la creación de la Secretaría Municipal de Educación en Derechos Humanos, las etapas del trabajo fueron integralmente realizadas, y las 
«metas da Prefeitura» en este ámbito fueron cumplidas en su totalidad, con destaque de los resultados objetivos y cuantitativos alcanzados, y el que éstos hayan sido muy productivos, junto con la incorporación en las leyes municipales de algunos de los legados de las políticas públicas.

Así, como balance, se considera que la Política Municipal de Educación en Derechos Humanos fue institucionalizada e introducida en los ámbitos más importantes en que su presencia era urgente y necesaria. Pero, posiblemente, la consolidación de las políticas públicas de educación en derechos humanos ocurrirá en una etapa posterior, a medida que hayan disminuido las primeras barreras y prejuicios en torno al tema, pues se sabe que los caminos en la educación en derechos humanos se encuentran mejor encauzados, situados y dirigidos, y se tiene presente que el objetivo para futuras iniciativas y políticas del área está mejor definido, conforme al plan de acción, por medio del documento normativo municipal que da forma al conjunto de conquistas para la ciudad de São Paulo en este campo: el Decreto 57.503/2016, que institucionaliza el Plan Municipal de Educación en Derechos Humanos (PMEDH). Queda claro que los avances y conquistas invitan a nuevas tareas futuras ${ }^{9}$ y que este documento puede servir de base para inspirar otras experiencias municipales, estatales y nacionales. Así, la experiencia de una megalópolis como la ciudad de São Paulo puede también fortalecer e inspirar la ejecución de experiencias en otros países.

Pero, teniendo presente el actual alto nivel de instabilidad, considerados el escenario de crisis económica y las dificultades políticas actuales del Brasil, es difícil anticipar cualesquiera que sean las perspectivas de futuro para la educación en derechos humanos.

\section{Referencias}

Benevides, Maria Victoria (2009). Fé na luta. São Paulo: Lettera.doc.

BitTAR, Eduardo Carlos Bianca (2013). Democracia, justiça e emancipação social. Reflexões jusfilosóficas a partir do pensamento de Jürgen Habermas. São Paulo: Quartier Latin.

-. (2016). «Os desafios de implementação das diretrizes nacionais para a educação em direitos humanos». En Gilberto Bercovici y otros (coordinadores), Desafios dos direitos humanos no século XXI, 163-176. São Paulo: Quartier Latin.

BuccI, Maria Paula Dallari (2002). Direito administrativo e políticas públicas. São Paulo: Saraiva, 2002.

Freire, Paulo (2013). Pedagogia da autonomia. Saberes necessários à prática educativa. 46. ${ }^{\mathrm{a}}$ ed. Río de Janeiro: Paz e Terra.

9. «Una vez más, y a base de insistir: avanzamos, y tenemos un largo camino por delante» (Benevides, 2009: 339). 
Schilling, Flávia (2014). Educação e direitos humanos. Percepções sobre a escola justa. São Paulo: Cortez.

\section{Apéndice: Otros recursos consultados}

Araújo, Ulisses Ferreira (2010). «Ética e direitos humanos na formação docente». En Lúcia de Fátima Guerra y otros (organizadores), Direitos humanos na educação superior. Subsídios para a educação em direitos humanos na Pedagogia, 327-337. João Pessoa: Editora Universitária da UFPB.

Barbosa e Melo, Vilma de Lurdes (2014). «Mídias e material didático no espaço escolar. A abordagem dos direitos humanos e diversidades no livro didático». En Elio Chaves Flores y otros (organizadores), Educação em direitos humanos e educação para os direitos humanos, 141-169. João Pessoa: Editora Universitária da UFPB.

Benevides, Maria Victoria. (2010). «Direitos humanos: desafios para o século XXI». En Rosa Maria Godoy Silveira y otros, Educação em direitos humanos. Fundamentos teórico-metodológicos, 335-350. Brasilia: Secretaría Especial de los Derechos Humanos de la Presidencia de la República.

-. (2015). Respeitar é preciso! Educação em direitos humanos. São Paulo: Instituto Vladimir Herzog; Secretaria Municipal de Direitos Humanos e Cidadania; Secretaria Municipal de Educação.

Bittar, Eduardo Carlos Bianca (2011). Democracia, justiça e direitos humanos. Estudos de teoria crítica e filosofia do direito. São Paulo: Saraiva.

-. (2014). «A experiência da Associação Nacional de Pesquisa e Ensino em Direitos Humanos (ANDHEP). Gestão, execução e representação». En Giuseppe Tosi y otros (organizadores), A formação em direitos humanos na educação superior no Brasil. Trajetórias, desafios e perspectivas, 375-396. João Pessoa-Paraíba: Editorial Universitária da UFPB.

-. (2016). «O procon municipal e a publicidade infantil». En Ana Estela Haddad (organizadora), São Paulo Carinhosa. O que grandes cidades e políticas intersetoriais podem fazer pela primeira infância, 413-421. São Paulo: Secretaria Municipal de Cultura.

Carbonari, Paulo César (coordinador) (2014). Educação em direitos humanos. Sistematização de práticas da educação básica. São Paulo, Passo Fundo: Município de São Paulo, IFIBE.

Comitê Nacional de Educação em Direitos Humanos (2013). Plano Nacional de Educação em Direitos Humanos. Brasilia, São Paulo: Secretaría de Derechos Humanos de la Presidencia de la República; Secretaría Municipal de Derechos Humanos y Ciudadanía; Unesco; MEC; MJ. 
Harvey, David (2014). Cidades Rebeldes: do direito à cidade à revolução urbana. São Paulo: Martins Fontes.

Prefeitura de São Paulo (2015). Respeitar é preciso! Educação em direitos humanos. São Paulo: Instituto Vladimir Herzog; Secretaría Municipal de Derechos Humanos y Ciudadanía; Secretaría Municipal de Educación.

-. (2014). $1^{\circ}$. Prêmio Municipal de Educação em Direitos Humanos da cidade de São Paulo. Relatos de experiência. 1. ${ }^{a}$ ed. São Paulo: Secretaría Municipal de Derechos Humanos y Ciudadanía; Secretaría Municipal de Educación.

-. (2015). $2^{\circ}$. Prêmio Municipal de Educação em Direitos Humanos da cidade de São Paulo. Relatos de experiência. 1. ${ }^{a}$ ed. São Paulo: Secretaría Municipal de Derechos Humanos y Ciudadanía; Secretaría Municipal de Educación, 2015.

-. (2016a). $3^{\circ}$. Prêmio Municipal de Educação em Direitos Humanos da cidade de São Paulo. Relatos de experiência. 1. ${ }^{\mathrm{a}}$ ed. São Paulo: Secretaría Municipal de Derechos Humanos y Ciudadanía; Secretaría Municipal de Educación.

-. (2016b). $4^{\circ}$. Prêmio Municipal de Educação em Direitos Humanos da Cidade de São Paulo. Relatos de experiência. 1. ${ }^{\mathrm{a}}$ ed. São Paulo: Secretaría Municipal de Derechos Humanos y Ciudadanía; Secretaria Municipal de Educación.

-. (2016c). Centros de Educação em Direitos Humanos da cidade de São Paulo. Territórios, educação e cidadania. São Paulo: Ciudad Escuela Aprendiz; Secretaría Municipal de Derechos Humanos y Ciudadanía, Secretaria Municipal de Educación. VAIner, Carlos (2013). "Quando a cidade vai às ruas». En Cidades Rebeldes. Passe livre e as manifestações que tomaram as ruas do Brasil, 35-40. São Paulo, Carta Maior: Boitempo.

\section{Sobre el autor}

Eduardo C. B. Bittar es profesor asociado del Departamento de Filosofía y Teoría General del Derecho de la Facultad de Derecho de la Universidad de São Paulo. Doctor en Filosofía y Teoría Derecho (1999) de la misma casa de estudios. Fue vicepresidente de la Asociación Brasileña de Filosofía del Derecho (ABRAFI-IVR). Fue coordinador de Educación en Derechos Humanos de la Secretaría Municipal de Derechos Humanos y Ciudadanía de São Paulo (SMDHC). Artículo traducido por Ana Bilbao. Su correo electrónico es edubittar@uol.com.br. 
